\title{
Criminologie
}

\section{En embuscade sur le sentier de l'argent. Une perspective internationale}

\section{Michael Levi}

Volume 30, numéro 1, printemps 1997

Criminalités économiques

URI : https://id.erudit.org/iderudit/017396ar

DOI : https://doi.org/10.7202/017396ar

Aller au sommaire du numéro

\section{Éditeur(s)}

Les Presses de l'Université de Montréal

ISSN

0316-0041 (imprimé)

1492-1367 (numérique)

Découvrir la revue

Citer cet article

Levi, M. (1997). En embuscade sur le sentier de l'argent. Une perspective internationale. Criminologie, 30(1), 35-52. https://doi.org/10.7202/017396ar
Résumé de l'article

This article examines the growth of financial measures against 'organised crime' in the form of money-laundering and asset confiscation. After discussing the implications of conflicts over what crimes should be included in money-laundering statutes - e.g. drugs-only or all 'serious' crime -it summarises the findings of a research study conducted by the author into the impact of money-laundering reporting in the UK upon criminal investigation by the police and customs, and into the forfeiture of the proceeds of crime. It concludes that these measures have had a very limited effect and will continue to do so, unless more technological and human resources are put into the investigation process. Furthermore, the tendency of offenders at all but the highest levels to spend their money as they go along places limits on the likely impact of these measures, based as they are on a model of criminal organisation that is more than the reality. 
This article examines the growth of financial measures against 'organised crime' in the form of money-laundering and asset confiscation. After discussing the implications of conflicts over what crimes should be included in money-laundering statutes -e.g. drugs-only or all 'serious' crime -it summarises the findings of a research study conducted by the author into the impact of moneylaundering reporting in the UK upon criminal investigation by the police and customs, and into the forfeiture of the proceeds of crime. It concludes that these measures have had a very limited effect and will continue to do so, unless more technological and human resources are put into the investigation process. Furthermore, the tendency of offenders at all but the highest levels to spend their money as they go along places limits on the likely impact of these measures, based as they are on a model of criminal organisation that is more than the reality.

\section{INTRODUCTION}

Au cours des années 1990, on s'est inquiété de la menace grandissante du crime organisé dans le monde, menace incarnée notamment par la « nouvelles » mafia russe de l'ex-U.R.S.S. et par les triades chinoises, redoutées jusqu'au Canada, même si le premier ministre chinois Dung Hsaio-Ping les décrivait comme des "groupes patriotiques »... Une bonne partie de cette nouvelle appréhension est suscitée par le trafic de la drogue ; l'extorsion, la contrebande et la fraude sont également préoccupants, et bien que le degré d'organisation de ces groupes criminels soit discutable, il y a fort à parier qu'une importante partie du commerce international se fait sur des valeurs mobilières d'origine illégale. Ce débat sur le crime organisé nous a amenés à nous concentrer sur un nouveau « démon », le blanchisseur de fonds, perçu comme un pivot du « système » criminel international et qui procure au crime organisé une voie d'accès à l'économie légitime ainsi qu'à certains avantages uniques qui engendrent une concurrence on ne peut plus déloyale.

1. Professeur de criminologie et directeur de White-Collar and Organized Crime Unit, School of Social and Administrative Studies, University of Wales, Cardiff, CF1 3AT, Wales, UK. 
Toutefois, si l'on considère de façon abstraite les dommages susceptibles d'être causés par la montée du crime organisé, à la lumière du discours officiel et des textes spécialisés de plus en plus nombreux à traiter du sujet, les groupes criminels se comporteraient différemment des présumés cartels ou corporations qui enfreignent la loi (Pearce et Snider, 1995 ; Snider, 1993). Il semble plausible que l'intégration économique de ces criminels mènera à une violence accrue ou, du moins, à une menace grandissante dans les pays où le gouvernement et le fisc se sont montrés plus fermes. Bien que l'examen de cette présomption soit une tâche très vaste à entreprendre, il vaut la peine de déconstruire cette perception du danger.

Comment les nations ont-elles réagi aux menaces, réelles ou prétendues, du crime organisé ? On a d'abord tenté de maîtriser le problème du crime organisé en isolant, d'une part, l'économie légitime et, comme Al Capone les appelait, les « rackets légitimes » (les comptables, les banquiers et les avocats) de la corruption d'autre part. Les développements politiques importants, les coûts d'implantations des normes et à la peur de la " criminalisation » parmi les professions libérales, du fait que leurs représentants et clients pourraient avoir des démêlés avec le système réglementaire sont devenus des lieux communs. D'une certaine façon, vu l'impopularité politique des banquiers, des comptables et des avocats, il a été difficile à ceux-ci de prouver qu'ils sont exempts de liens avec le crime organisé, ce, d'autant qu'ils font circuler des fonds pour les trafiquants de drogues. Aussi une nouvelle pratique est-elle apparue, qui vise à contrecarrer l'activité des organisations criminelles par la confiscation de leurs avoirs. Quelques-unes des mesures visant à enrayer le blanchiment de fonds ont été harmonisées, entre autres grâce à l'influence d'organismes tels que le Financial Action Task Force (FATF, mis sur pied par les pays du G-7, fortement influencés par le Département d'État des États-Unis). Le Canada fut un des premiers pays à commander des études sur les méthodes de blanchiment de fonds (Beare, 1996 ; Beare et Schneider, 1990 ; Francis, 1988), mais cela n'a pas eu d'effet d'entraînement sur les mesures de contrôle au plan international. Nourrissant des doutes à la fois sur le coût et sur l'efficacité du système américain de déclaration obligatoire des activités de change, le FATF a recommandé l'adoption du système anglais, moins coûteux, en vertu duquel les banques sont tenues (ou du moins autorisées) de transgresser les règles de confidentialité en signalant les cas suspects aux autorités. (Les États-Unis ont maintenant adopté ce système sans pour autant abandonner leur système de déclaration.) Les mesures législatives prévues pour contrôler les revenus d'origines criminelles n'ont pas encore été uniformisées à l'échelle mondiale parce que - la limitation des actifs mis à part — ce sont là des mesures post facto, non préventives, et parce que les risques que courent les services financiers d'un pays ayant adopté des lois de confiscation draconiennes ne 
sont pas les mêmes que ceux pris par les services financiers de pays ayant adopté des mesures anti-blanchiment beaucoup plus coûteuses et lourdes que celles de leurs concurrents. De plus, le fardeau des mesures anti-blanchiment incombe en majeure partie au secteur privé (sauf pour ce qui concerne les coûts des enquêtes policières, relativement peu élevés), tandis que la confiscation des avoirs relève de l'État. D'autre part, au plan international, les pays ne sont pas tous d'accord sur le type de crimes visés par la loi antiblanchiment : des conflits importants ont vu le jour entre ceux qui (ÉtatsUnis en tête, mais aussi la Grande-Bretagne et le Canada) désirent que la réglementation sur le blanchiment de fonds soit appliquée à tous les crimes (y compris la fraude dans les pays où elle fait l'objet et de poursuites) et ceux qui (comme la Chine, le Japon et la Russie) souhaitent que ces lois ne portent que sur les profits du trafic de stupéfiants. Ce qui est en jeu jci, c'est la crainte que chaque État soit forcé de cesser de blanchir l'argent qui donne lieu à de l'évasion fiscale chez l'autre. Cette question pourrait être soulevée lors des sommets internationaux, au chapitre du principe international bien établi qui veut que les pays ne sont pas tenus d'appliquer les lois fiscales des autres et également (bien que ce ne soit pas discuté ouvertement) la question de la peur des élites que le financement politique fasse l'objet d'une réglementation trop sévère quant aux transferts financiers. Compte tenu des révélations de 1995 et de 1996, sur les fonds secrets en Corée du Sud - plus de 700 millions de dollars par président - et du grand intérêt des Japonais pour les dons occultes, les inquiétudes générées par l'impact d'un éventuel enrayage du blanchiment doivent effectivement être vives.

\section{ÉVALUATION DE LA PERFORMANCE ANTI-BLANCHIMENT}

On s'est beaucoup moins préoccupé, sauf verbalement, des résultats éventuels que peuvent atteindre les systèmes de contrôle destinés à contrôler le blanchiment d'argent (de la confiscation également) et du moyen d'évaluer leurs performances. Une description simple et claire de la mise en place éventuelle de mesures anti-blanchiment dans ces sphères privées que constitue le monde financier nous fait encore défaut.

Dans cette perspective, en 1992, mon associé de recherche, Michael Gold et moi-même, avons entrepris d'évaluer l'effet des contrôles sur le blanchiment d'argent au Royaume-Uni avant que ne soient introduites les Lois sur le blanchiment de fonds en 1993 (Gold et Levi, 1994). L'évaluation d'un effet est toujours problématique. D'abord, comment peut-on estimer l'effet dissuasif des lois sur le blanchiment d'argent si on n'a aucune idée de la somme d'argent qui est blanchie ? Ensuite, comment peut-on juger des effets possibles des mesures anti-blanchiment sur le blanchiment lui-même ainsi que sur la distribution et la consommation de stupéfiants, dès lors que l'effort 
réel de lutte contre celles-ci peut être négligeable ou très variable selon les institutions financières et les forces policières en cause ? Et finalement, comment peut-on juger comme dans toute politique de " maintien de l'ordre » de l'efficacité à long terme de certains facteurs tels que les «bonnes relations » entre les corps policiers et les institutions financières, qui peuvent avoir des résultats difficiles à mesurer durant la période d'évaluation ? Aucun de ces problèmes n'appelle de solution simple, mais il est tout de même important de les identifier. Faute de temps et d'argent, nous avons opté pour des entrevues et des observations en profondeur de quatre des plus importantes banques à charte au Royaume-Uni et avons réalisé des entrevues avec un échantillon ad hoc (des contacts obtenus de bouche à oreille) de compagnies d'assurances et de banques commerciales. Nous nous sommes également servis des conclusions d'un résumé détaillé que j'avais moi-même réalisé en 1988-1990 sur les relations entre banques et corps policiers (Levi, 1991). Nous avons échantillonné un cas sur dix, sur un total de 1000 cas rapportés en 1991 à l'organisme alors connu sous le nom de National Drugs Intelligence Unit (devenu depuis le National Criminal Intelligence Service, ou NCIS), afin de connaître les résultats des rapports relatifs aux cas ayant été transmis à la police ou aux douanes pour une enquête plus approfondie. (Nous avons étudié un échantillon supplémentaire de 300 cas, en 1993, afin de déterminer s'il y avait eu des changements significatifs, en nous attardant particulièrement aux cas de « réussite », décrits par le NCIS, dans le but d'en identifier les caractéristiques communes.) J'ai longuement interviewé des officiers de police et de douanes aux spécialités diverses (fraude, drogues, terrorisme) à Londres et dans les provinces anglaises, en Écosse, au pays de Galles et en Irlande du Nord, afin de connaître l'usage qu'ils faisaient des divulgations financières et de savoir à quels problèmes ils étaient confrontés. J'ai également tenté de voir si les rapports des institutions financières les avaient aidés lors d'enquêtes criminelles majeures. Au Royaume-Uni et dans la plupart des pays d'Europe, à quelques différences près, c'est l'institution financière qui prend la décision de faire un rapport au bureau central de police ; celui-ci (le NCIS, au Royaume-Uni) transmet le rapport bancaire aux services de police et de douanes compétents, après quoi les enquêteurs des régions concernées décident du lieu, du moment et de l'ampleur de l'enquête. J'ai finalement interrogé des enquêteurs et des représentants fédéraux nordaméricains, australiens et européens sur le mode de fonctionnement de leurs systèmes anti-blanchiment. (La démarche de recherche sur la confiscation était similaire ; elle comporte un examen exhaustif du déroulement des enquêtes de certaines unités ainsi qu'un questionnaire pour les autres.) 


\section{RÉSULTATS DE RECHERCHE}

Les résultats de cette recherche sont essentiellement des données statistiques et des descriptions qui ne prennent toute leur signification qu'à la lumière du contexte dont ils proviennent, soit les systèmes financiers, juridiques et judiciaires. Le premier élément à souligner est notre conclusion, basée non seulement sur des entrevues, mais aussi sur l'hypothèse $a$ priori qu'il y a tendance à surestimer la somme d'argent blanchi, en la mettant sur le même pied que les profits du crime. Sauf dans les plus hautes sphères, (où ils ne peuvent pas toujours être dépensés et où leur entreposage physique serait trop encombrant et risqué, les profits criminels sont, pour la plupart, affectés à la consommation ostentatoire des criminels eux-mêmes, de leurs familles et de leurs amis, plutôt qu'à l'épargne ou aux investissements. Les criminels peuvent disposer d'un surplus d'argent venant du trafic de stupéfiants, et donc avoir des problèmes temporaires d'entreposage, mais ce n'est que lorsque le volume des profits (net, après les dépenses en hommes de main et autres frais d'opération) devient trop élevé pour être dépensé dans l'immédiat que les criminels font face à des problèmes de blanchiment. C'est souvent par les dépenses que des criminels font lors de leurs voyages et leurs loisirs, faisant affaire avec des bookmakers, casinos, concessionnaires d'automobiles, propriétaires de clubs, gérants de restaurants, lignes aériennes, ou faisant effectuer des travaux de rénovation ou la décoration à domicile que les profits criminels entrent dans le système bancaire officiel. En conséquence, on a souvent tendance à surévaluer le blanchiment dont font l'objet les profits criminels, quoiqu'on ne puisse nier que les criminels ont monétairement bénéficié de leurs crimes.

Les ressources financières allouées aux enquêtes ayant été limitées, les rapports sur les transactions suspectes effectués par les banques, sociétés de crédit immobilier, et autres entités réglementées (au nombre de 18000 en 1994, continuant d'augmenter au Royaume-Uni et maintenant 60 fois plus nombreux qu'au départ), fournissent rarement des renseignements susceptibles de permettre aux policiers et aux douaniers de mettre sur pied une opération de surveillance d'un criminel particulier. Cela dit, l'information permet de constituer des dossiers, et plusieurs rapports sur la même personne, ou sur ses acolytes, peuvent inciter à mener une enquête plus détaillée. Par ailleurs, dans la plupart des cas, il s'agit de personnes « connues » dans le milieu ou déjà sous surveillance. En raison de la faible proportion de dénonciations qui débouchent sur une enquête approfondie plutôt que sur une simple vérification sur les réseaux d'information criminelle, nous ne pouvons évaluer la proportion des dénonciations qui, si elles donnaient lieu à un sérieux suivi, permettraient d'établir des preuves d'activité criminelle. De plus, bon nombre de cas relevant du Serious Fraud Office au Royaume-Uni (tels 
ceux de Maxwell et Polly Peck et celui, plus prévisible vu qu'il implique une banque, de la $\mathrm{BCCl}$ ) de même que les scandales qui ont éclaté au Canada et en France démontrent la facilité avec laquelle les grandes sociétés peuvent transférer des fonds outre-mer sans éveiller de soupçon d'activité criminelle ni, en cas de soupçon, susciter de dénonciation auprès du UK National Criminal Intelligence Service (NCIS) - ou auprès de services analogues - ni même déclancher des poursuites contre les présumés bénéficiaires ayant été dénoncés.

Jusqu'en 1994, la pratique consistant à déclarer des transactions intercompagnies faisait totalement défaut au sein du système de détection du blanchiment de fonds ; c'est encore là un sujet d'avenir, puisque aucun pays n'a encore mis sur pied de « système d'éveil de soupçon » pour ces transactions, tout ce qui a été fait se limitant à la formulation de quelques platitudes comme : « surveillez les compagnies dont les entrées d'argent ne correspondent pas « au volume des ventes » (qui d'ailleurs n'est pas forcément connu). Il est en outre difficile pour les banquiers ou pour tout autre intervenant de surveiller de telles transactions faute de temps et d'argent. Le personnel des banques étant victime de coupures, une telle surveillance pose des difficultés financières.

Bien que les plus récentes données démontrent que ces chiffres sont à la hausse, jusqu'ici peu de dénonciations (environ 4 sur 1000) ont déclenché de nouvelles enquêtes ou ont eu un effet significatif sur des enquêtes déjà en cours, notamment en empêchant un criminel de haut vol d'échapper à une condamnation. Néanmoins, ces dénonciations ont bel et bien donné lieu à des poursuites (songeons par exemple à celles qui avaient trait à la pratique très répandue chez les employés de la Banque d'Angleterre de voler des billets de banque hors des "cages de destruction », au détournement de fonds d'un comptable chargé des dossiers d'opérations financières clandestines à Scotland Yard et à quelques violations des principales règles bancaires. Dans quelques-unes des récentes causes où de grandes quantités de billets de banques ont été mises à jour dans les résidences de trafiquants de stupéfiants appréhendés, il semble que les activités des malfaiteurs s'en sont trouvées perturbées en se sens qu'ils se sont vus obligés d'entreposer de grosses sommes d'argent pour des périodes plus longues. Toutefois on ne peut pas s'attendre à ce que les banquiers connaissent le type de crime, s'il y a crime, en se basant uniquement sur le comportement d'un client suspect. De plus, on ne doit pas voir en ce ratio de 4 dénonciations sur 1000 , cité plus tôt, 4 cas avérés de blanchiment d'argent sur 1000 ; il ne s'agit que de soupçons, et à moins qu'on fasse enquête, ce qu'on ne fait pas, les institutions financières ne peuvent que rarement garantir qu'il en aille de profits d'origine criminelle. 
Bien que cela soit moins vrai en Australie qu'au Royaume-Uni et au Canada, les systèmes de détection semblent avoir été conçus sans qu'on ait tenu compte des pratiques quotidiennes des corps policiers et douaniers dans les cas de transactions douteuses. À cet égard, la législation sur le blanchiment d'argent peut être perçue comme l'aboutissement symbolique d'un processus législatif mi-fonctionnel, mi-emblématique, même si elle est d'abord destinée à prévenir le blanchiment, à le limiter et plutôt qu'à le poursuivre à fournir des renseignements d'ordre comptable sur lui.

\section{LA SAISIE ET LA CONFISCATION DES AVOIRS : L'EXPÉRIENCE DU ROYAUME-UNI}

Le deuxième outil du "nouveau maintien de l'ordre " dans le monde financier c'est la confiscation des avoirs. En Angleterre et au pays de Galles, la théorie qui fonde la confiscation veut que celle-ci empêche le criminel de tirer un avantage financier d'un comportement social inacceptable ; elle ne relève pas de la dimension punitive de la sentence, ne l'atténue pas ní ne l'aggrave. Actuellement, la confiscation, qui est d'un intérêt croissant pour les « rétributivistes » permet, aux termes du Proceeds of Crime Act de 1995, (déjà en vigueur) de mettre la main sur les biens de tout criminel nouvellement condamné - pas seulement pour trafic de drogue - qui ne parvient pas à démontrer au tribunal qu'ils ont été acquis de façon légitime. Toutefois, quelque soit le statut Héonique de la confiscation en droit, les gens impliqués dans les enquêtes sur les profits đu crime voient la confiscation comme une mesure qui frappe vraiment les criminels :

- en les privant des bénéfices monétaires qu'ils convoitent le plus

- en minant leur crédibilité dans le milieu criminel.

Le criminel qui réussit à convaincre ses codétenus que "ses 》 avoirs l'attendent à sa sortie de prison gagne un certain prestige aux yeux de ceuxci ; à l'inverse, celui qui est publiquement dépouillé de ses avoirs perd ce prestige. Il n'y a aucune donnée relative au sexe au Royaume-Uni. Cela dit, par des entrevues auprès de la police et des enquêtes, on sait que presque tous les criminels sérieux commettant ce genre de crimes sont de sexe masculin. Et il en va de même au Canada.

Une autre justification théorique de la confiscation est son effet dissuasif présumé. Si on arrive à convaincre les criminels que « le crime ne paie pas » et que, s'ils se font prendre, ils ne pourront conserver les avoirs acquis grâce au crime, certains délinquants seront peut-être dissuadés de commettre des actes illégaux. Certaines de mes entrevues avec des criminels m'ont porté à croire que plusieurs d'entre eux considèrent les profits du crime comme leur « dû » ; leur confisquer ce dû les frustrerait naturellement et serait perçu 
comme une punition. De prime abord, toutefois, il semble n'y avoir aucune raison de s'attendre à ce que la confiscation amène ces individus à ne plus commettre d'actes criminels. Elle pourrait bien les déterminer encore davantage à obtenir leur « dû » quitte à ce que, comme au jeu de serpents et échelles » (= de l'oie), ils s'exposent à se retrouver subitement à la case « départ ». Par ailleurs, si, comme le croyons les corps policiers et moi-même, une bonne part des profits criminels est dépensée avant l'arrestation et, $a$ fortiori, avant la confiscation, les effets dissuasifs, punitifs et réparatifs peuvent être négligeables. Finalement, dans la prétendue guerre contre le crime organisé (distincte de celles contre le crime financier ou politique), on peut estimer que la « saisie des avoirs » (Canada et États-Unis) ou la « confiscation » (Royaume-Uni) est de nature à paralyser l'organisation en lui enlevant son moteur financier, en éliminant donc sa capacité d'échange et en la rendant moins attrayante aux yeux d'éventuelles recrues. Alors que si l'on accepte le modèle du crime désorganisé (Reuter, 1983) l'efficacité prévisible est plus problématique et l'on en est alors réduit à l'effet dissuasif sur les seuls individus.

Pour ces raisons à la fois philosophiques et pragmatiques, l'idée d'empêcher de « tirer profit du crime » est un leitmotiv important auquel ont adhéré les pays industrialisés anglophones au cours de la dernière décennie, et c'est là une idée qui s'est répandue en Europe depuis la convention européenne sur le blanchiment de fonds et la confiscation des profits criminels de 1991 . Cette idée offre aussi la possibilité de rendre certains domaines d'enquête policière financièrement autonomes, bien que cela n'ait jamais été le but avoué de la loi sur la confiscation en Angleterre et au pays de Galles, l'on considère généralement que la constitution informelle, dénie au gouvernement le droit de créer à même ses revenus des postes de dépenses spéciales.

Nonobstant le désintérêt quasi total des chercheurs britanniques (voir Ashworth 1993, bien que Thomas, 1992,1993, fasse exception), les principes fondant la saisie ont fait l'objet de débats passionnés en Australie (Fisse et coll., 1992) de même que dans les cercles universitaires et judiciaires américains. Dans Halper, $109 \mathrm{~S}$. Ct. 1902, la Cour suprême a jugé que le but curatif de la saisie était insuffisant pour justifier son caractère punitif. (Voir aussi les commentaires du juge Scalia dans Austin v. États-Unis (1993) $113 \mathrm{~S}$. Ct. 2801). On s'est demandé aux États-Unis si la saisie des avoirs enfreignait l'article sur les « peines excessives » du $8^{\mathrm{e}}$ amendement (fondé sur la Déclaration anglaise des droits de 1689). Cette question revêt une certaine importance si l'on tente de régler les cas affectés des plus nettes disproportions, mais, jusqu'ici, l'impact de cet amendement a été négligeable. Pourtant, au Royaume-Uni, on ne se préoccupe pas des principes formels, sauf de ceux qui sont imposés par la Convention Européenne des droits de l'homme. 


\section{L'ESTIMATION DES PROFITS CRIMINELS}

Avant de se pencher sur le processus de confiscation et sur ses effets, il peut être utile de chercher à savoir combien d'argent est susceptible d'être confisqué. La somme à considérer se divise en deux : (i) le revenu total du crime ; (ii) la part de ce bénéfice susceptible d'être confisquée. Pour connaître, cette part, il faut pouvoir évaluer les habitudes de dépense et d'économie des criminels, très mal connues. L' « économie criminelle » alimentée par des revenus illicites, peut si l'on exclut l'évasion fiscale, être divisée en deux catégories : (a) les crimes dits «sans victimes » ou de racket : le crime est commis par consentement mutuel (comme le trafic de stupéfiants ou la prostitution) ou il consiste en une extorsion qui n'est pas rapporté à la police : (b) les crimes contre la propriété en vue d'un gain, sous forme d'argent ou de biens qui sont soit volés soit obtenus frauduleusement. L'estimation de la somme d'argent générée par le trafic de stupéfiants et les crimes de catégorie (a) est plutôt floue, en raison de la difficulté d'évaluer les dommages subis par les entreprises et les familles. Par contre, au vu de certaines suppositions plus précises concernant les profits que réalisent les criminels sur la revente de leurs biens volés (b) on estime à environ 1,6 milliards $£$ la somme des profits criminels obtenus autrement que par des fraudes visant des services financiers et des faillites (voir plus loin Levi et Osofsky, 1995).

On ne peut que faire des conjectures sur la part de cet argent qui est économisé plutôt qu'utilisé pour acquitter le coût des dépenses courantes et pour impressionner les amis et les voisins, mais rien ne permet de croire que les criminels font beaucoup d'épargne. Les recherches américaines et britanniques démontrent plutôt qu'ils sont de gros consommateurs qui dépensent leur argent au fur et à mesure ; il n'y a aucune raison de croire que la situation est différente ailleurs, pas davantage au Canada. De plus, une bonne partie de cet argent serait disponible pour des indemnisations, mais pas celui, selon l'interprétation qu'on peut donner du Proceeds of Crime Act de 1995, dont on ne peut prouver ni l'acquisition légitime, ni le rattachement à une victime particulière. Ceci peut affecter les revenus gouvernementaux générés par la confiscation, mais non les bénéfices du processus en lui-même : pour calculer le bénéfice net, on doit déduire la somme recouvrable par des poursuites civiles plus celle qui aurait effectivement été accordée par un juge, du total obtenu par les ordres de confiscation les plus draconiens.

Par ailleurs, si on considère le trafic de stupéfiants, on constate que l'habituelle hypothèse des « dix pour cent », selon laquelle les douaniers interceptent 10 pour cent de tous les stupéfiants entrant au Canada et aux ÉtatsUnis, est purement arbitraire, surtout lorsqu'on sait que ce pourcentage serait constant, indépendamment de la période ou du type de stupéfiant. Le produit brut des ventes d'héroïne aux simples usagers et aux revendeurs 
usagers a été évalué à un total de 600 millions $£$ en 1990 . Au cours de notre récente étude sur le blanchiment de fonds, (Gold et Levi, 1994), nous avons estimé que les profits liquides qui sont déposés dans des institutions financières du Royaume-Uni pour être blanchis (et pour être ensuite partiellement réintégrés dans l'économie légitime) et qui sont donc sujets à la confiscation pourraient ne pas dépasser la somme de 100 millions $£$ par année, même si elle est également susceptible d'être beaucoup plus importante. On doit ajouter à ces montants les propriétés, voitures, bateaux et tout autre avoir vendable acquis avec des profits du trafic de stupéfiants ou tout autre " service » de corruption, vice ou racket ; cependant on n'ajoute pas les services totalement consommés en comme les paris, le tourisme, etc., dans lesquels les criminels dépensent leur argent.

En conclusion, même selon une estimation prudente des profits du crime en Angleterre et au pays de Galles, les sommes dont la confiscation est ordonnée, abstraction faite de celles qui sont bel et bien confisquées, semblent être proportionnellement très faibles. Cela s'explique, en partie, assez facilement: selon les dispositions législatives antérieures, la confiscation ne pouvait porter que sur des profits de criminels incarcérés pour trafic de stupéfiants ou (selon une législation maintenant révolue) sur ceux de quelques criminels non-trafiquants qui étaient condamnés une première fois pour des crimes dont le bénéfice total était supérieur à $10000 \mathfrak{f}$. Comme très peu de ces criminels importants ont été condamnés, leurs profits n'ont pu être saisis (sauf dans des cas comme celui du vol de lingots du Brink's Mat du RoyaumeUni, où des poursuites civiles « ordinaires », régies par des normes de preuve plus lâches, ont été entamées contre des auteurs acquittés ou non-poursuivis). Pour résumer, il est plausible qu'il y ait près de 460 millions $f$ de profits tirés de crimes contre la propriété et (selon une estimation prudente) au moins 100 millions $f$ de profits issus du trafic de stupéfiants qui seraient confiscables par année. En Angleterre et au pays de Galles, de 1987 à décembre 1995, 7478 jugements de confiscation ont été rendus, en majorité pour trafic de stupéfiants, pour un total de près de 100 millions $f$, dont 62 millions $f$ pour des cas de douanes. Les données du service des procureurs de la Couronne ne sont pas systématiquement mises à jour, mais on sait que les douanes ont récupéré $23 \%$ du total exigé, et $15 \%$ supplémentaires sur les comptes bloqués en attente d'appel. En nous basant sur l'hypothèse raisonnable que la cour n'est pas plus apte à faire appliquer les ordres de confiscation non-douaniers que les douaniers, un ma-ximum de 25 millions $\mathfrak{f}$ a été confisqué sur une période de 8 ans, toutes sources confondues.

\section{COMPARAISON DES SYSTÈMES BRITANNIQUE ET AMÉRICAIN}

Puisque une bonne partie de l'aggiornamento du processus de confiscation vient des États-Unis et que plusieurs Britanniques interviewés au cours 
de nos recherches (Levi et Osofsky, 1994, 1995) semblaient envier ce qu'ils prenaient, souvent à tort, pour la réalité américaine, jl importe de noter l'énorme différence entre l'échelle des saisies civiles et criminelles de biens aux États-Unis et celle de l'Angleterre. (Des discussions informelles suggèrent que certains Canadiens éprouvent les mêmes envies.) La somme totale des saisies perçues par le Département du Marshall aux États-Unis était de 3275,8 millions de dollars sur une période de temps un peu plus longue, que celle qui va de 1985 à septembre 1993 ; elle atteignait 555,7 millions de dollars pour la période de janvier à septembre 1993.

En d'autres termes, le Marshall des États-Unis obtient effectivement $140 £$ pour chaque $1 £$ obtenu en Angleterre et au pays de Galles. Les recettes de 1993 dépassaient à elles seules d'au moins 20 fois le total des recettes de l'Angleterre et du pays de Galles depuis 1987. Les chiffres nets de 1993 des États-Unis sont supérieurs au total brut de l'Angleterre depuis 1987. Certains autres contrastes sont révélateurs. Le sondage de Levi et Osofsky (1995) a dénombré environ trois cas, à l'échelle du pays en 1993, où des entreprises ont fait l'objet de saisies pour une confiscation effective ou projetée. L'État fédéral américain avait 4499 propriétés et entreprises sous saisie en 1993, valant 859 millions $\$$ au total. Au cours de l'exercice se terminant en septembre 1994, le Département de justice américain a encaissé le montant record de 3,1 milliards $\$$ dans le cadre de poursuites civiles et criminelles, alors qu'il n'a perçue que 1,5 milliards \$ en 1993 et 1,7 milliards $\$$ en 1992. Les perceptions en argent liquide s'élevaient à 1,83 milliards $\$$, perçues à titre d'amendes, de taxes spéciales, de frais de justice, de recouvrements de prêts et de recouvrements prévus par le False Claims Act ; les perception non liquides qui s'élevaient à 1,28 milliards $\$$ prenaient la forme de transferts de propriété, de paiements faits à des offices et des cours ne relevant pas du Département de justice, et de compensations imputées sur des sommes que le gouvemement aurait rayées autrement.

Toutefois, on s'est montré sceptique quant à l'impact en terme d' « offre " de la confiscation sur les criminels. Karchmer (1985, p. 39) souligne :

" $0,0062 \$$ out of every illegally earned dollar from narcotics traffic is subject to the initiation of some type of government removal action. This figure loses further significance when one looks at the outcomes of various removal actions: less than half the amounts involved in seizure and forfeiture actions were actually surrended to the government, and no more than 2 per cent of IRS jeopardy and termination assessments (which totalled $\$ 81.3$ million in 1981) ended up being collected. In the light of these statistics, the value of asset removal strategies is highly questionable." 
Les poursuites intentées contre les criminels en col blanc et les organisations corrompues peuvent avoir quelque peu modifié favorablement le rendement - et certainement les sommes nettes et brutes récupérées - depuis leur introduction, mais l'argument principal de Karchmer relatif au faible ratio des saisies par rapport aux profits criminels conserve sa validité et remet en question l'effet de la saisie sur les ventes de stupéfiants. Ceci est particulièrement vrai lorsqu'on examine les données sachant qu'on ne peut qu'estimer les sommes d'argent blanchies au États-Unis, au Royaume-Uni, en Australie et au Canada et que ces sommes sont probablement déjà surestimées.

Les sommes plus élevées, que recouvrent les États-Unis s'expliquent par certains facteurs que voici :

(I) l'intérêt pour le crime en col blanc des autoritês qui savent pouvoir trouver plus d'avoirs chez les gens des professions libérales ou d'autres individus dont les biens sont plus stables et les habitudes de consommation moins extravagantes;

(II) un recours plus fréquent aux enquêtes à long-terme qui visent les groupes du crime organisé et les réseaux qui y sont associés ;

(III) des enquêtes approfondies sur les membres des professions libérales (avocats, comptables, courtiers) qui sont les bras droits des trafiquants et qui sont susceptibles de posséder plus d'avoirs ;

(IV) le Racketeer Influenced Corrupt Organization Act de 1970 (RICO) et autres dispositions sur le crime organisé lesquelles facilitent l'imposition de lourdes pénalités lors de poursuites judiciaires civiles ou criminelles ;

(V) la possibilité qu'ont les autorités américaines d'utiliser les pouvoirs (dits in rem) de saisie civile. Ces pouvoirs permettent de saisir les avoirs mal acquis sans qu'une condamnation criminelle soit prononcée. Au Royaume-Uni, on ne peut avoir recours à la confiscation sans qu'une condamnation criminelle ait été rendue pour un délit quelconque.

En revanche, on peut dire que la saisie à l'américaine des avoirs a involontairement des conséquences négatives. D'abord, elle détourne l'attention des enquêteurs criminels des individus dépensiers pour la porter sur des citoyens marginalement criminels tels les membres des professions libérales, qui possèdent plus d'avoirs susceptibles d'être saisis et qui se font prendre par une législation très imprécise. Deuxièmement, cette procédure peut mener à un excès de zèle et à une violation des libertés civiles de la part des policiers, 
qui, depuis que la confiscation de véhicules chics ou puissants peut contribuer à satisfaire les besoins des officiers de police, se découvrent un intérêt personnel au sein d'un système qui dépend institutionnellement pour sa propre survie des avoirs saisis (ou, du moins, pour les ressources techniques qu'on lui alloue). Le choix des cibles à saisir peut parfois sembler arbitraire et indicatif, quoique de récents jugements rendus en appel aient insisté sur l'idée de proportionnalité entre le délit et la saisie. Les effets secondaires négatifs semblent plus notables dans les corps policiers municipaux et provinciaux qu'au sein des forces fédérales. À cela s'ajoute le risque permanent que la corruption réduise artificiellement la somme des produits mais c'est là un risque connu de tous les systèmes judiciaires).

Il est difficile d'évaluer l'impact de la confiscation sur les contrevenants et les « organisations criminelles » - une expression souvent ambiguë, comme les corps policiers s'en sont aperçus - avec lesquelles certains contrevenants s'associent. Une des raisons du peu d'effet global de la confiscation sur les individus et les groupes, du moins au Royaume-Uni, c'est que, quand les biens d'un individu sont confisqués, cet individu peut se replacer dans une organisation. En effet, lorsque l'individu quitte la prison, s'il a une position favorable parmi ses collègues - il est à noter que la confiance ou la peur sont d'importantes conditions préalables au succès criminel tout comme dans les affaires légitimes - il peut réintégrer l'organisation et acquérir des biens, comme de la drogue, à crédit. Même le criminel seul qui agit pour son propre compte semble, au vu d'éléments anecdotiques sur les criminels récidivistes, n'avoir besoin que de très peu d'avoirs pour retourner à ses activités criminelles, après un séjour en prison et la confiscation de ses avoirs. En conséquence, un individu ne sera pas «sorti » des affaires par une confiscation même si, cornme après une faillite personnelle, il devra réduire l'ampleur de ses activités pour une période plus ou moins longue. La raison qui sous-tend prioritairement la confiscation n'a jamais été de mettre un terme aux activités des criminels individuels. Une autre opinion, des plus désabusées, est que la confiscation, suite à un premier crime ne fait que rendre le criminel plus prudent en vue de la commission d'un second crime. Aussi, le criminel de niveau moyen se montre plus apte à camoufler ses avoirs après une première confiscation; les criminels de plus haut vol prévoient l'éventualité d'une confiscation de leurs avoirs dès le premier vol !

La conclusion selon laquelle les criminels apprennent à éviter les erreurs ne vaut pas pour tous les criminels. Certains peuvent être trop idiots pour retirer une quelconque leçon de leurs expériences, tandis que d'autres peuvent ne pas avoir de compréhension suffisante ni les contacts adéquats leur permettant de disposer de leurs avoirs de façon plus subtile. D'autres encore 
peuvent simplement ne pas vouloir abandonner le crime. Plusieurs continueront donc à faire ce qu'ils ont toujours fait soit dépenser leur argent à mesure, adopter un mode de vie hédoniste où l'épargne (et la confiscation) a très peu d'importance. Ceci n'exclut pas la possibilité d'un certain impact sur l'individu visé. Ainsi, les grossistes fournissant à crédit de la drogue aux revendeurs peuvent se retrouver en fâcheuse posture des suites de la saisie des biens d'un revendeur. Ce dernier est alors exposé à des représailles. L'opinion de la plupart des policiers est que la confiscation a trop peu d'effets sur la criminalité en général, malgré l'impact relatif qu'elle peut avoir sur des individus.

De façon générale, les policiers reconnaissent à la confiscation une influence perturbatrice sur les fonds de roulement des criminels de moyen et bas niveaux en ce sens qu'elle force ces derniers à recommencer au bas de l'échelle, en s'exposant aux risques de condamnation plus élevés qui sont reliés au commerce de la rue. La confiscation frappe durement les criminels, nuit à leur réputation aux yeux de leurs pairs et représente l'un des seuls moyens d'obtenir de leur part des renseignements sur leurs activités, quand ils veulent éviter de perdre des biens dont les autorités connaissent l'existence. Cette façon officieuse de négocier était perçue par les policiers comme une tactique raisonnable à employer au cours d'une enquête, mais elie est également devenue une source d'inquiétude quant au rôle joué par les procureurs ou les avocats lorsqu'ils décident, en négociant, du traitement qui est réservé à d'importants efforts d'investigation.

\section{CONCLUSION : ÉVALUER LA SIGNIFICATION DU NOUVEAU MAINTIEN DE L'ORDRE}

Le Royaume-Uni s'est vanté de son succès dans l'implantation d'un système efficace d'auto-contrôle et de coopération entre corps policiers et banques, auprès de pays étrangers, affirmant entre autres qu'une des plus grandes forces du système britannique est la relation positive de travail entre autorités policières et institutions financières. Par ailleurs, la citoyenneté active, qui confère aux institutions financières un rôle social de premier plan, est un élément positif pouvant se traduire en « capital social ». La collaboration des institutions financières avec les corps policiers et le législateur dans le développement de lignes directrices pour l'interprétation des règles applicables a conféré une plus grande légitimité aux réglementations. Elle est aussi à l'origine de plus de cohérence notamment grâce à la formation assez uniforme sous l'égide du British Bankers' Association. Par conséquent, l'implantation du système britannique a produit un certain bénéfice politique et judiciaire, même si, pour les sceptiques, c'est tous les pays, que les banquiers ont fait en sorte que soit adoptée et appliquée une loi qui ne rende pas leurs 
activités trop difficiles, particulièrement en ce qui a trait aux affaires « corporatives ». Bien qu'il y ait très peu de condamnations pour blanchiment de fonds, et ce spécialement quand il en va de membres des professions libérales, la Banque d'Angleterre et les autres organes régulateurs exercent un contrôle informel et réglementaire (comme au Canada et dans les autres pays dont l'économie est developpée) ; il ne faut pas penser que le petit nombre de poursuites démontre qu'il y a très peu d'activitê criminelle, ni le considérer comme un indicateur d'inaction régulatrice. Les données portent à sousestimer la véritable efficacité du système d'enquête britannique. En effet, dans les années à venir, je m'attends à ce que plusieurs individus et sociétés ayant été identifiés comme suspects et n'ayant encore jamais fait l'objet de poursuites seront inspectés de façon plus approfondie s'ils refont parler d'eux. D'où toute la difficulté d'une évaluation à court terme.

Il y a véritablement problème lorsqu'une société capitaliste s'attend à ce que les banques évaluent en profondeur l'origine morale de l'argent ce media le plus anonyme et le plus international - ne serait-ce que dans les cas d'attaques à l'ordre public. Toutefois, la valeur des réglementations sur le blanchiment d'argent devrait être évaluée eu égard aux difficultés rencontrées par les enquêteurs chargés de dossiers criminels d'jmportance. Vu ces difficultés assez répandues (songeons à l'identification des criminels, à la surveillance, au développement et l'utilisation de contacts d'information ou de délation, surtout lorsqu'il y a une crise financière qui crée de plus grandes attentes en termes d'efficacité) toute source d'information noncorrompue et désintéressée compte, particulièrement lorsque le renseignement est proactif (soit, lorsqu'il est obtenu, en principe avant que la police n'apprenne la nouvelle d'un crime ou que l'individu dont la conduite est rapportée ne soit soupçonné de méfaits). Les opérations bancaires clandestines sont restées largement hors de portée de la réglementation de l'économie formelle on peut au contraire soutenir qu'elles ont été stimulées par ces contrôles et par la chute de la BCCI - mais une certaine habileté de la part des agences policières a permis d'examiner, par le biais de l'accès aux dossiers financiers avant le déroulement de procédures criminelles, ce que trament les sous-cultures de la drogue asiatique et afro-antillaise, difficilement pénétrables autrement. Néanmoins, il faut plus de réalisme dans l'appréciation de l'impact de ces changements. Théoriquement, les transactions douteuses rapportées par les banquiers offrent la possibilité de cibler le crime de manière impartiale, sur la base d'une analyse objective de réseau de type neuronal. En pratique, les banquiers usent des mêmes indices de soupçon que la police comme ceux ayant trait aux comportements hors-contexte.

McQueen (1994) et Fraser (Fisse et coll, 1992, pp. 49-50), font tous deux preuve de scepticisme, affirmant : 
Maintenant, au lieu de bombarder la piste [...] d'Ho Chi Minh, nous devons nous en prendre à la piste de l'argent par saisies, confiscation, infraction de blanchiment d'argent, soit par un bombardement massif dans la guerre contre les stupéfiants... Rien de plus complexe qu'une guerre pour le contrôle du marché... Dans certains cas, c'est un conflit dont l'enjeu est l'existence même d'un marché (par exemple, le marché noir de la drogue); dans d'autres, il s'agit simplement de tenter de réglementer les activités, afin de s'assurer que le marché demeure neutre et juste, dans son fonctionnement.

Pour Fraser, l'objectif semble être d'éliminer le profit du crime dans le but de rendre le crime non rentable. Toutefois il y a mieux : il y eut une tendance, au moins réthorique, à chercher à se servir des institutions financières proactivement de façon à obtenir de l'information pour :

a) d'identifier les individus qui pourraient être impliqués dans des crimes et qui n'étaient pas soupçonnés auparavant. (Évidemment, on pourrait englober dans ce groupe les dissidents politiques, mais je ne crois pas qu'il s'agisse là d'un objectif-clé) ;

(b) de fournir une preuve plus complète de certaines activités de criminels connus, preuve normalement trop coûteuse en temps et en argent pour les moyens traditionnels d'enquête dont disposent les policiers.

Une partie de cette tâche peut être effectuée par ordinateur, mais les ressources policières ne reflètent guère l'importance attachée en principe à cette tâche : un enquêteur peut devoir mener jusqu'à six enquêtes de routine par jour, soit 30 par semaine et certains peut-être 1200 par année, enquêtes qui ne consistent qu'en une vérification succincte des éventuels antécédents du suspect. Le NCIS est complètement submergé par le travail réactif de ce type et les forces de police britanniques font face au même problème : certains policiers ne consultent même pas leurs banques de données locales.

Il n'est pas du tout certain que ces aspects du new-policing puissant être qualifiés de rhétoriques, et ni que la législation qui en découle soit symbolique. J'ai suivi les relations entre corps policiers et banques, depuis la mise en place de cette législation, et je dirais que les plus grandes banques se montrent inquiètes en songeant à l'implantation de celle-ci et aux risques d'emprisonnement. Elles ont dépensé près de 30 millions $f$ annuellement en coûts d'implantation au Royaume-Uni. Mais la motivation et les dépenses pourraient baisser lorsque les banques réaliseront qu'il y a très peu de poursuites judiciaires qui sont intentées grâce à leur contribution et qu'il est peu probable qu'elles soient elles-mêmes poursuivies ou sanctionnées par la Banque d'Angleterre pour mauvais comportement. Il est également difficile d'évaluer la peur qu'inspirent les sanctions, au Royaume-Uni, du Financial 
Action Task Force, une unité supranationale (bien qu'officiellement temporaire) de régulateurs habilités à examiner et commenter la satisfaction des pays membres du système. Toutefois, la crainte «corporative " et gouvernementale de telles sanctions (y compris la stigmatisation) peut avoir certains effets, indépendamment de la probabilité que ces sanctions soient prononcées. L'hypothèse que de telles mesures auront un effet significatif sur le trafic de stupéfiants ainsi que sur la consommation — c'est là l'objectif théorique de contrôle de l'offre - est d'autant plus discutable, qu'un individu qui s'y connaît en affaires est en mesure de trouver des moyens « intercorporatifs » pour effectuer des transferts financiers difficiles à détecter, et ce indépendamment de ce qu'est le système de blanchiment. Les nouvelles approches technologiques telles que les réseaux neuronaux et les systèmes exploitant l'information disponible sont susceptibles d'être utilisées pour détecter et contrer le blanchiment autrefois indétectable. Toutefois, contrairement à la fraude par carte de crédit, par exemple, où de tels systèmes sont utilisés depuis un certain nombre d'années (Levi et coll. 1991), les circonstances entourant le blanchiment et son ampleur sont très peu connues, ce qui rend difficile le développement d'un système d'identification commode ne produisant pas trop de fausses pistes et n'effectuant pas trop de recherches onéreuses sur des transferts monétaires s'avérant finalement légitimes. De telles recherches mobjlisent plus de personnel que l'État et les banques, qui n'ont pas d'intérêt direct à ce que la détection se passe, ne sont prêts à payer. Les criminels à col blanc, quant à eux, comme dans d'autres domaines de leurs activités, disposent d'un avantage par rapport à ce qu'il en est avec d'autres crimes tendant à un enrichissement ; la composante centrale des systèmes anti-blanchiment, soit l'humain est, en effet, plus à son aise avec les transferts monétaires manuels qu'avec les transferts de fonds électroniques d'un compte « corporatif » apparemment légitime à un autre.

\section{BIBLIOGRAPHIE}

ASHWORTH, A. (1992), Sentencing and Criminal Justice, Londres : Weidenfeld \& Nicholson. AUDIT Commission (1993), Helping with Enquiries, Londres : HMSO.

BEARE, M. (1996), Criminal Conspiracies : Organized Crime in Canada. Scarborough, Ont. : Nelson Canada.

BEARE, M. et SCHNEIDER, S. (1990), Tracing of Illicit Funds : Money-Laundering in Canada, Ottawa : Solliciteur-Général du Canada.

DUYNE, P. van (1994), 'Estimates in Fog' Journal of Asset Protection, 2(1), 58-76.

Financial Crimes Enforcement Network (1992), An Assessment of Narcotics-Related Money Laundering, Washington, D.C. : U.S. Treasury.

FISSE, B., FRASER, D. et COSS. G. (eds) (1992), The Money Trail, Sydney : The Law Book Co. FRANCIS, D. (1988), Contrepreneurs, Toronto : Macmillan.

GOLD, M. et LEVI, M. (1994), Money Laundering in the U.K : An Appraisal of Suspicious Transaction Reporting, London: Police Foundation. 
KARCHMER, C. (1985), 'Money Laundering and the Organized Underworld', in H. Alexander et G. Caiden (eds.) The Politics and Economics of Organized Crime, Lexington : Lexington Books.

LEVI, M. (1981), The Phantom Capitalists : The Organisation and Control of Long-Firm Fratd, Aldershot: Gower.

LEVI, M. (1991), Customer Confidentiality; Money Laundering, and Police-Bank Relationships, London : Police Foundation.

LEVI, M. (1993), The Investigation, Prosecution and Trial of Serious Fraud. Royal Commission of Criminal Justice Research Study $n^{\circ} 14$, London : HMSO.

LEVI, M., BISSELL, P. and RICHARDSON, T. (1991). The Prevention of Cheque and Credit Card Fraud, Crime Prevention Unit paper 26. London : Home Office.

LEVI, M. and OSOFSKY, L. (1994). 'Confiscating the Proceeds of Crime Around the World: Some Issues for Bankers' in R. Parlour (ed.) The International Handbook of MoneyLaundering, London : Butterworths.

LEVI, M and OSOFSKY, L. (1995) Investigating, Seizing and Confiscation the Proceeds of Crime, Police Research Group Paper 61, London : Home Office.

LEVI, M. and PITHOUSE, A (forthcoming), Victims of White-Collar Crime, Oxford : Oxford University Press.

PEARCE, F. and SNIDER, L. (eds.) (1995), Corporate Crime : Contemporan Debates, Toronto : University of Toronto Press.

McQUEEN, Row (1994) 'Your Money and Your Life', ANZ Journal of Criminology, 27, 174-91. REUTER, P. (1993), Disorganized Crime, Cambridge, Mass : MIT Press.

SNIDER, L. (1993), Bad Business : Corporate Crime in Canada, Scarborough, Ont. : Nelson Canada.

SUTTON, M. and MAYNARD, A. (1992), What is the Size and Nature of the 'Drug' Problem in the U.K. ?, Yartic Occasional Paper, 3, York : Centre for Health Economics.

SUTTON, M. and MAYNARD. A. (1994), Trends in the Cost-Effectiveness of Enforcement Activity in the llicit Heroin Market, 1979-1990. Yartic Occasional Paper 4, York : Centre for Health Economics.

THOMAS, D. (1982), Current Sentencing Practice, updated loosedleaf, London : Sweer \& Maxwell.

THOMAS, D. (1994), 'The Criminal Justice Act 1993: (1) Confiscation Orders and Drug Trafficking', Criminal Law Review, 93-100. 